\title{
Influence du niveau d'entretien des parcelles de cacaoyers sur la prévalence de la pourriture brune des cabosses à Kipiri, Sud-Ouest de la Côte d'Ivoire.
}

\author{
Franck Zokou Oro ${ }^{* \pm^{1}}$, Hermann-Desiré Lallie ${ }^{2}$, Mohamed Doumbouya ${ }^{1}$, Juslin Koigny ${ }^{1}$ and Hortense \\ Atta Diallo 3 \\ 1Département de biologie végétale, Unité de Formation et de Recherche (UFR) des Sciences Biologiques, Université \\ Peleforo GON COULIBALY. BP 1328 Korhogo \\ 2Département de biochimie-génétique, Unité de Formation et de Recherche (UFR) des Sciences Biologiques, \\ Université Peleforo GON COULIBALY. BP 1328 Korhogo. \\ 3Unité de Recherche en Phytopathologie, Département de Protection de Végétaux et de l'Environnement, Université \\ Nangui Abrogoua.
}

Original submitted in on 19th November 2019. Published online at www.m.elewa.org/journals/ on $31^{\text {st }}$ December 2019 https://doi.org/10.35759/JABs.144.10

\section{RESUME}

Objectif: L'objectif principal de cette étude était de déterminer l'influence du niveau d'entretien des parcelles de cacaoyers sur la prévalence de la pourriture brune des cabosses. II s'agira d'évaluer la dépendance du niveau d'entretien des parcelles sur la progression de cette maladie.

Méthodologie et résultats : Une expérimentation a eu lieu dans des plantations paysannes sur un site sentinelle à Kipiri de $10 \mathrm{Km} \times 10 \mathrm{Km}$ divisé en 16 Clusters de 2,5 Km × 2,5 Km. Cette expérimentation a permis de recueillir des données du niveau d'entretien des parcelles et d'incidence, ainsi que de la cartographie de la pourriture brune des cabosses du cacaoyer. Le niveau d'entretien des parcelles est une variable qualitative à deux modalités (Entretenue et Non Entretenue). Les résultats du test d'ANOVA à 1 facteur ont montré que la prévalence de la pourriture brune des cabosses est dépendante du niveau d'entretien de la parcelle. Cette étude a permis d'identifier deux espèces de Phytophthora sp dont Phytophthora megakarya et Phytophthora palmivora dans des proportions respectives de $46 \%$ et $28 \%$. Contrairement aux expériences précédentes, cette étude montre que Phytophtora megakarya est l'espèce la plus répandue sur ce site. II ressort que la recrudescence de la maladie est fortement liée à la forte dispersion de cette espèce qui est la plus virulente.

Conclusion et application des résultats: Ces résultats obtenus permettent de faire plusieurs recommandations aux producteurs de cacaoyers. Des récoltes sanitaires et des désherbages réguliers doivent être faits pour éliminer les sources de l'inoculum dans les parcelles. De même, les tailles régulières des cacaoyers doivent être réalisés pour permettre une bonne aération de la parcelle, brisant ainsi les conditions adéquates de développement des pathogènes.

Mots clés : Cacaoyer, niveau d'entretien des parcelles, pourriture brune des cabosses, Soubré, Côte d'lvoire. 


\section{ABSTRACT}

Objectives: The main objective of this study was to determine the influence of maintenance level of cocoa plots on the prevalence of black pod disease. It will assess the dependence of maintenance level of the plots on the progression of this disease.

Methodology and results: An experiment was carried out in peasant plantations on a sentinel site in Kipiri of $10 \mathrm{Km} \times 10 \mathrm{Km}$ divided into 16 Clusters of $2.5 \mathrm{Km} \times 2.5 \mathrm{Km}$. This experiment allowed to collect plots relative data of maintenance level and black pod disease. The maintenance level of plots is a qualitative variable with two modalities (Maintained and Non-Maintained). The results of the 1-way ANOVA test showed that the prevalence of black pod disease is dependent on the maintenance level of the plots. This study identified two species of Phytophthora sp including Phytophthora megakarya and Phytophthora palmivora in respective proportions of $46 \%$ and $28 \%$. Unlike previous experiences, this study shows that Phytophtora megakarya is the most widespread species on this site. It shows that the upsurge of disease is strongly related to the strong dispersion of Phytophthora megakarya which was the most virulent specie.

Conclusion and application of results: These results make it possible to make several recommendations to cocoa farmers. The first step will be for them to carry out regular sanitary harvesting to eliminate the sources of inoculum in the plots. Secondly, regular weeding will be done to destroy the probable nests of insects and fungal pathogens. Thirdly to practice the regular size of cocoa trees to allow a good aeration of the plot, thus breaking the adequate conditions of pathogens development.

Key words: Cocoa tree, plot maintenance level, black pod disease, Soubré, Côte d'Ivoire.

\section{INTRODUCTION}

Le cacaoyer (Theobroma cacao L.) est un arbre de la famille des Malvaceae cultivé dans de nombreux pays de la zone intertropicale d'Amérique, d'Afrique et d'Asie du Sud-est (Babin, 2009). En Côte d'Ivoire, la production annuelle de cacao est passée de 900.000 tonnes en 1995 à 1.500 .000 tonnes en 2011 (Marius et Foluke, 2015). Cette augmentation est liée à une expansion de la zone de culture de cacao qui a commencé dans les années 1970, quand la production de cacao s'est déplacée du Sud-Est vers le Sud-Ouest (Marius et Foluke, 2015). La Côte d'Ivoire produit $40 \%$ de cacao à l'échelle mondiale, ce qui fait d'elle le premier producteur au monde (Dufour, 2005). L'exploitation du cacao représente $40 \%$ des recettes d'exportation et contribue à hauteur de 10 $\%$ du Produit Intérieur Brut (PIB) (Tano, 2012). Cependant, la culture du cacaoyer est confrontée à de nombreuses maladies virales (Swollen shoot) et fongiques dont l'une des principales est la pourriture brune des cabosses. La pourriture brune des cabosses est provoquée par un champignon parasite du genre Phytophthora qui occasionne d'importantes pertes de rendement (Coulibaly et al., 2013). Phytophthora sp existe sous quatre principales espèces dont $P$. palmivora, $P$. megakarya; $P$. capsici et $P$ citrophthora (Coulibaly et al., 2013). Parmi ces espèces, seules $P$. palmivora et $P$. megakarya constituent les espèces les plus importantes en Côte d'Ivoire (CCC, 2015). Les pertes de récoltes occasionnées par ces deux espèces, s'élèvent à environ $30 \%$ de la production mondiale (Ndoumbe, 2002) et varient de 20 à 45 $\%$ en Côte d'Ivoire (Coulibaly et al., 2017). Ces pertes augmentent le taux de pauvreté des producteurs de cacao de 9,7\% (Ouattara, 2015).

Par ailleurs, des travaux réalisés par Gidoin (2013) au Centre du Cameroun ont révélé que les parcelles non entretenues favorisent le développement de la pourriture brune des cabosses et l'installation des insectes ravageurs.

L'entretien des parcelles inclue le désherbage régulier et la taille régulière des cacaoyers pour éliminer les nids des pathogènes fongiques et les insectes susceptibles de disperser la pourriture de cabosses (CCC, 2015). De plus, les études de Coulibaly et al. (2013) à l'Est, au Centre-Ouest et au Sud-Ouest des plantations ivoiriennes ont permis de montrer que $P$. palmivora est l'espèce la plus dominante du verger ivoirien. Ces études restent encore insuffisantes car, la prévalence de la pourriture brune des cabosses du cacaoyer est 
toujours d'actualité. La lutte contre cette maladie se base sur l'utilisation des produits phytosanitaires dont les fongicides (Coulibaly et al., 2017) qui ont un impact négatif sur l'homme et l'environnement. Pour pallier à cela, il est important d'identifier les facteurs de pratiques culturales à l'origine de la forte prévalence de la pourriture brune des cabosses en Côte d'lvoire, et notamment dans la principale zone de production dont Soubré. Ce travail vise à étudier l'impact du

\section{MATERIEL ET METHODES}

Site d'étude: Le site d'étude est situé à Kipiri (Soubré), au Sud-Ouest de la Côte d'Ivoire (Figure 1) à 6.14 degrés de latitude Nord et à -6.46 degré de longitude Ouest avec une altitude de 242 mètres. La zone est caractérisée par un climat subéquatorial (Koua, 2018) dominé par deux saisons pluvieuses et deux saisons sèches: une grande saison de pluies comprise entre avril et juin et une petite saison pluvieuse entre septembre et novembre. Les deux saisons sèches se situent entre juillet et août et entre niveau d'entretien des parcelles de cacaoyers sur la prévalence de la pourriture brune des cabosses à Kipiri. De façon spécifique, il s'est agi de :

- Identifier et cartographier la distribution des espèces Phytophthora $s p$ responsables de la pourriture brune des cabosses.

- Etudier la relation entre la prévalence de la pourriture brune des cabosses et le niveau d'entretien des plots (parcelles).

décembre et mars. La pluviométrie moyenne oscille entre $1600 \mathrm{~mm}$ et $1800 \mathrm{~mm}$ et les températures varient entre $26^{\circ} \mathrm{C}$ et $32^{\circ} \mathrm{C}$ (Koua, 2018). Le sol de la zone d'étude est fertile avec une végétation marquée par la forêt dense et humide qui fait place aujourd'hui à d'immenses plantations de cultures industrielles de cacao, de café, d'hévéa et de palmier à huile. Cette zone est donc riche en potentialités agricoles et forestières.

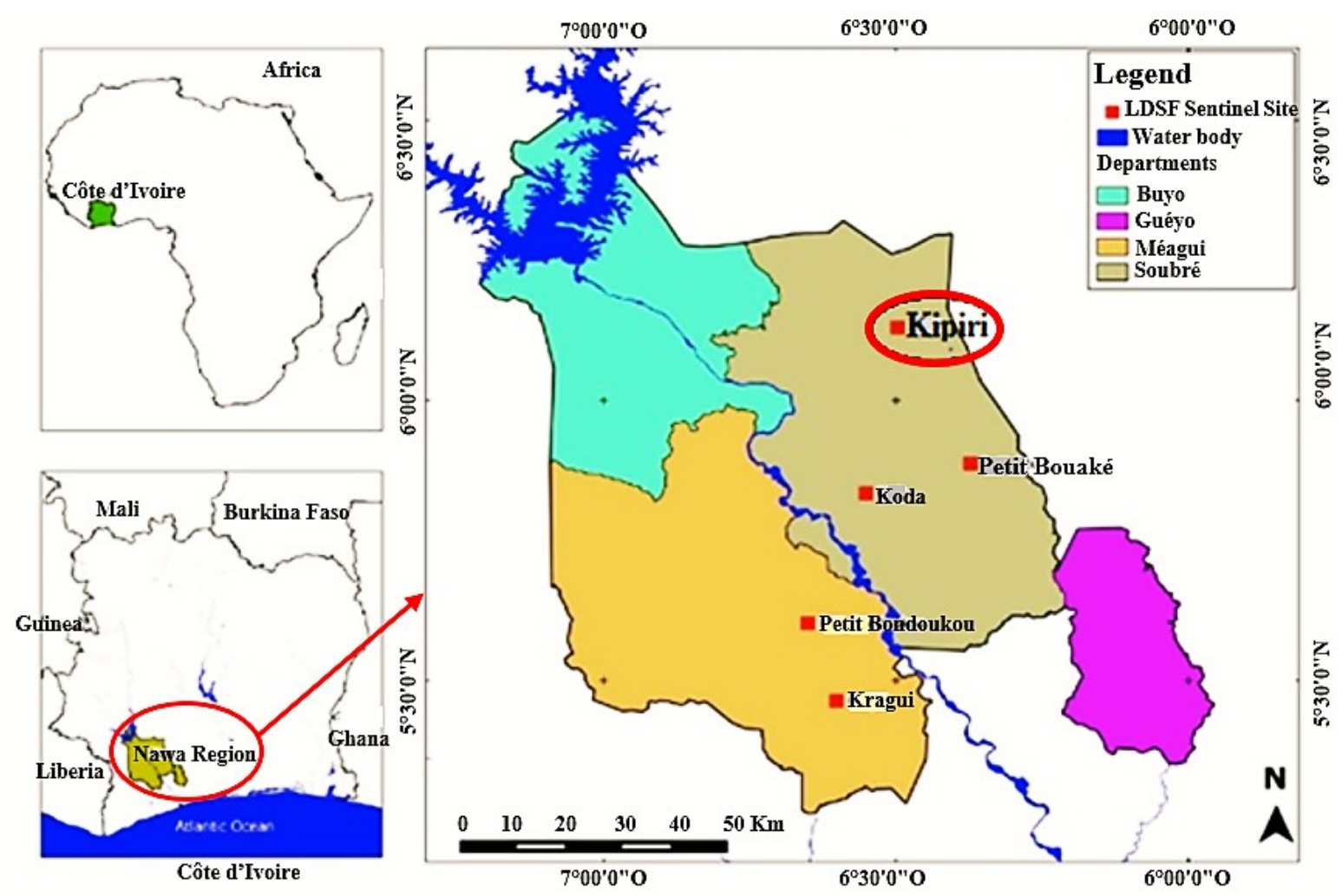

Figure 1 : Carte de la région du Nawa présentant le département de Soubré qui comprend le site de Kipiri (Diby et al., 2014) 
Dispositifs de collecte de données : La collecte de données a été réalisée suivant le dispositif LDSF (Land Degradation Surveillance Framework) dans des plantations paysannes (Diby et al., 2014). Ce dispositif LDSF a été initialement mis au point par ICRAF à Nairobi pour évaluer et suivre les sols propices à l'agriculture en Afrique de l'Ouest. Cependant, il a été adapté pour la collecte des données relatives aux pathologies végétales (Diby et al., 2014). Le LDSF représente un site sentinelle de $10 \mathrm{Km} \times 10 \mathrm{Km}$. Ce site est divisé en 16 clusters de 2,5 $\mathrm{Km} \times 2,5 \mathrm{Km}$ (Figure 2). Chaque cluster comprend 160 points d'observation, appelés plots. Dans le cas de cette étude 65 plots ont été échantillonnés. Ces plots ont été choisis au hasard. Les données des pratiques culturales dont le niveau d'entretien des plots (parcelles), ainsi que les données épidémiologiques relatives à la pourriture brune des cabosses telles que le nombre de cabosses pourries, le nombre total de cabosses ont été collectées dans un rayon de 50 mètres autour du centre de chaque plot d'échantillonnage (Figure 3).

Identification moléculaire et cartographie des espèces de Phytophthora $s p$ : Après un diagnostic sur la base des symptômes de la pourriture brune au champ, un prélèvement de petits morceaux de cortex de cabosses pourries est effectué à partir d'une coupe transversale. Ces explants de cabosses sont conditionnés dans des tubes eppendorf en milieu favorable à Phytophthora sp. Chaque cacaoyer échantillonné a été géolocalisé avec le GPS Garmin. Au laboratoire, les explants échantillonnés ont été cultivés en boîte de Pétri sur le milieu "V8", qui se compose d'un 1/10 de cocktail de jus de légumes, gélose à $15 \mathrm{~g} / \mathrm{L}$ et $\mathrm{CaCO} 3$ à $3 \mathrm{~g} / \mathrm{L}$ pendant 4 jours dans l'obscurité, à $25^{\circ} \mathrm{C}$. Après 4 jours, les implants d'agar contenant du Phytophthora mycelium ont été retirés du front de croissance fongique avec un scalpel puis repiqués dans le milieu V8 dans les mêmes conditions décrites ci-dessus pendant 7 jours. Le mycélium résultant est utilisé pour l'extraction d'ADN. Dans le cas où les souches sont contaminées par d'autres champignons, plusieurs transplantations sur milieu de culture $\mathrm{H} 2 \mathrm{O}-$-Agar à $15 \mathrm{~g} / \mathrm{L}$ ont été nécessaires pour purifier la souche. Après purification, la souche est à nouveau cultivée sur le milieu "V8" à $25^{\circ} \mathrm{C}$ pendant 4 jours puis 7 jours à l'obscurité pour obtenir un mycélium typique de Phytophthora sp. Le mycélium obtenu après purification est utilisé pour l'extraction d'ADN. L'identification des différentes espèces de Phytophthora a été réalisée par PCR (Polymerase Chain Reaction) avec des amorces ITS spécifiques à chaque espèce ( $P$. megakarya et $P$. palmivora).

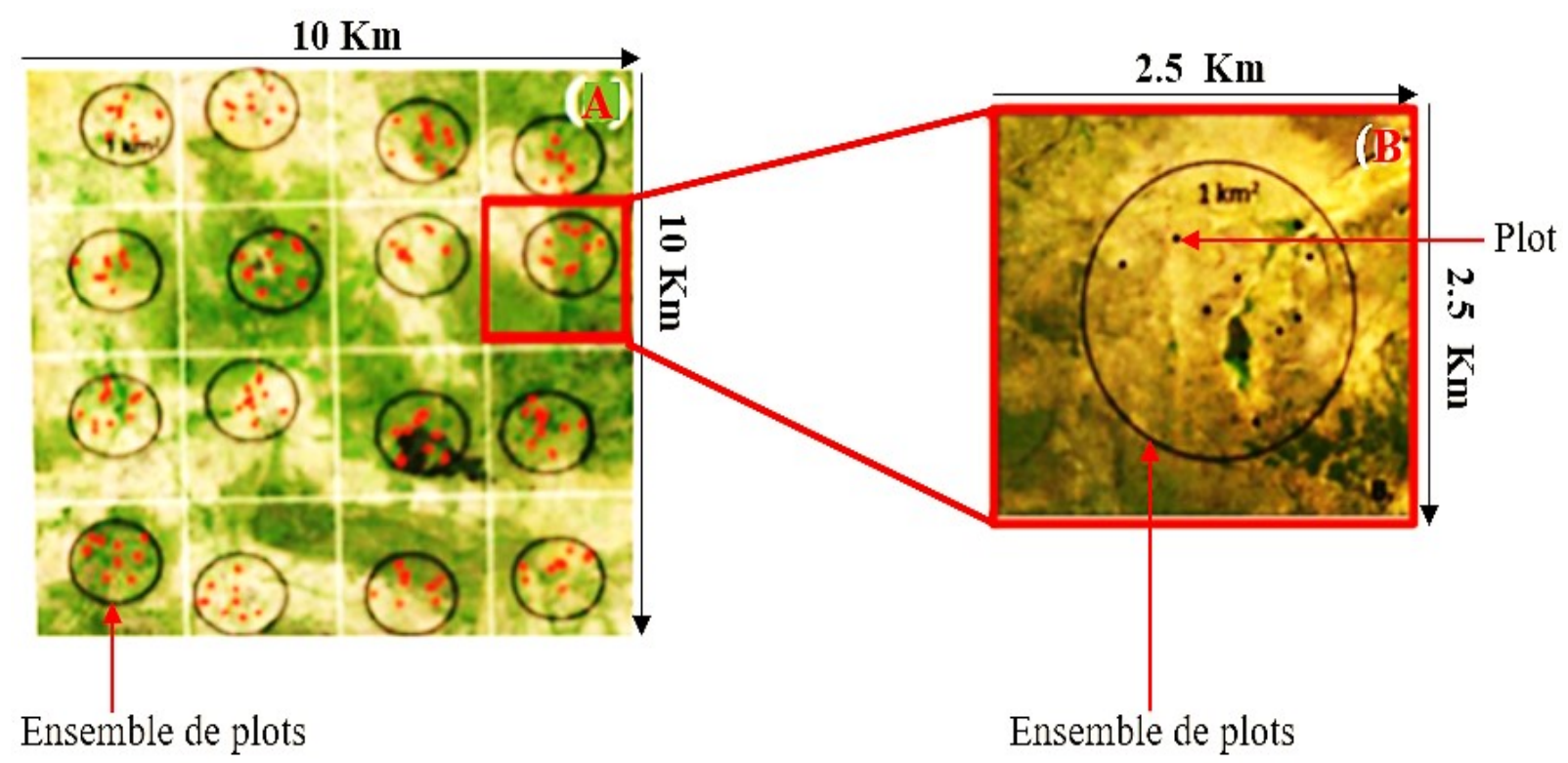

Figure 2: Dispositif Land Degradation Surveillance Framework (Diby et al., 2014) 


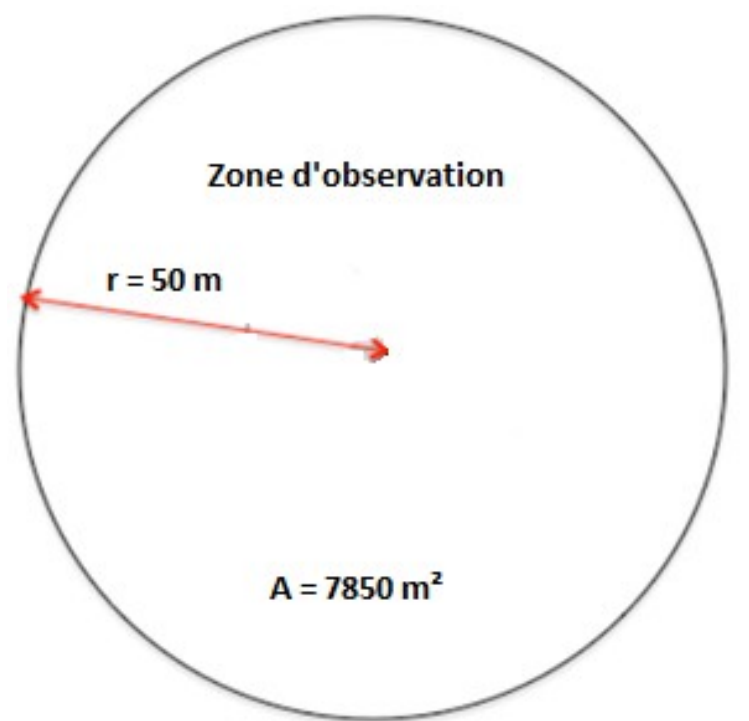

Figure 3 : Plot avec l'aire d'observation (Diby et al., 2014)

Observation et collecte de données : Soixante-cinq (65) plots géolocalisés et repartis sur 16 clusters ont été échantillonnés. Ces plots ont permis de récolter les données relatives à Phytophthora $s p$ et les données relatives au niveau d'entretien (NE) des parcelles.

Collecte de données du Niveau d'Entretien (NE) des plots (parcelles) : Les données du Niveau d'Entretien des parcelles ont été collectées dans un rayon de $50 \mathrm{~m}$ autour du centre du plot d'observation. Le NE des plots est une variable qualitative à deux modalités dont la modalité « non entretenu » qui a été notée zéro (0) et la modalité «entretenu» notée un (1). Le plot non entretenu est un plot qui n'a pas fait l'objet d'un désherbage et d'un égourmandage récents au moment de l'observation, contrairement au plot entretenu.

Données épidémiologiques et prévalence de phytophthora sp: Les données épidémiologiques constituées du nombre total de cabosses (T-Cab) et du nombre total de cabosses atteintes de la pourriture brune des cabosses (I-Cab). Ces données ont été relevées sur au moins 10 cacaoyers portant la pourriture brune des cabosses dans chaque plot. La prévalence de la pourriture brune des cabosses du cacaoyer représente le ratio du nombre total de cabosses infectées (I-Cab) par rapport au nombre total de cabosses (T-Cab). Cette prévalence est déterminée par la formule suivante :

Prévalence de la pourriture brune $=\frac{\mathrm{I}-\mathrm{Cab}}{\mathrm{T}-\mathrm{Cab}} \times \mathbf{1 0 0}$

Analyse de données: L'Analyse des données a d'abord porté sur la cartographie de distribution des espèces de phytophthora déjà identifiées. En effet, les résultats issus de l'identification moléculaire des espèces de phytophthora $s p$ ont été utilisés pour produire les cartes de répartition de $P$. palmivora et de $P$. megakarya à partir des coordonnées géographiques des arbres échantillonnés. Cette carte a été produite à partir du logiciel IBM SPSS Statistics version 20. Ensuite, les analyses statistiques ont porté sur la description des paramètres relatifs au niveau d'entretien et de la prévalence de la pourriture brune des cabosses. De plus les boxplots ont permis de comprendre la dispersion des données de prévalence de la maladie et leur répartition selon le niveau d'entretien. Le test de comparaison des moyennes ANOVA à un facteur a permis de comparer la prévalence de la pourriture brune des cabosses dans chacune des modalités de la variable niveau d'entretien. 


\section{RESULTATS ET DISCUSSION}

Cartographie des différentes espèces de Phytophthora sp: Les résultats de l'analyse d'identification ont permis de réaliser la carte de répartition des espèces de Phytophthora sp sur le site de Kipiri (Figure 4). Ces résultats ont montré que $P$. megakarya est plus dispersé sur l'ensemble des zones d'observation avec une fréquence de $46 \%$ que $P$. palmivora et les autres espèces de Phytophthora qui ont en moyenne une fréquence de $27 \%$ (Figure 5).

Relation entre le NE des parcelles et la prévalence de la pourriture brune des cabosses : La description des données relatives à la prévalence de Phytophthora $s p$ (Tableau 1) a donné une moyenne de 22,65\% $\%$ 6,3 sur l'ensemble des plots tests des 16 clusters échantillonnés. Cela montre que les données de la prévalence de Phytophthora sp sont regroupées autour de la moyenne. Les plots non entretenus ont représenté $69 \%$ de tous les plots tests contre 31\% pour les plots entretenus (Tableau 2). La Figure 6 représente les boxplots de la prévalence de Phytophthora en fonction du Niveau d'Entretien des plots. Les résultats ont montré que les données de prévalence dans les plots non entretenus sont plus dispersées par rapport à celles dans les plots entretenus. La moitié $(50 \%)$ des observations sur les plots non entretenus ont une prévalence comprise entre 25 et $32 \%$. Tandis que plus de la moitié des plots entretenus ont une prévalence se situant entre 18 et 20 $\%$. Les résultats du test de ANOVA à un 1 facteur ont montré qu'il existe une relation significative $(P=0,018$ $<0,05)$ entre le Niveau d'Entretien des plots d'observation et la prévalence de la pourriture brune des cabosses (Tableau 3). Ce qui révèle que la prévalence de la pourriture brune des cabosses du cacaoyer est dépendante du Niveau d'Entretien des parcelles. Les plots non entretenus ont une prévalence de la pourriture brune significativement plus élevé que celles observés dans les plots entretenus.

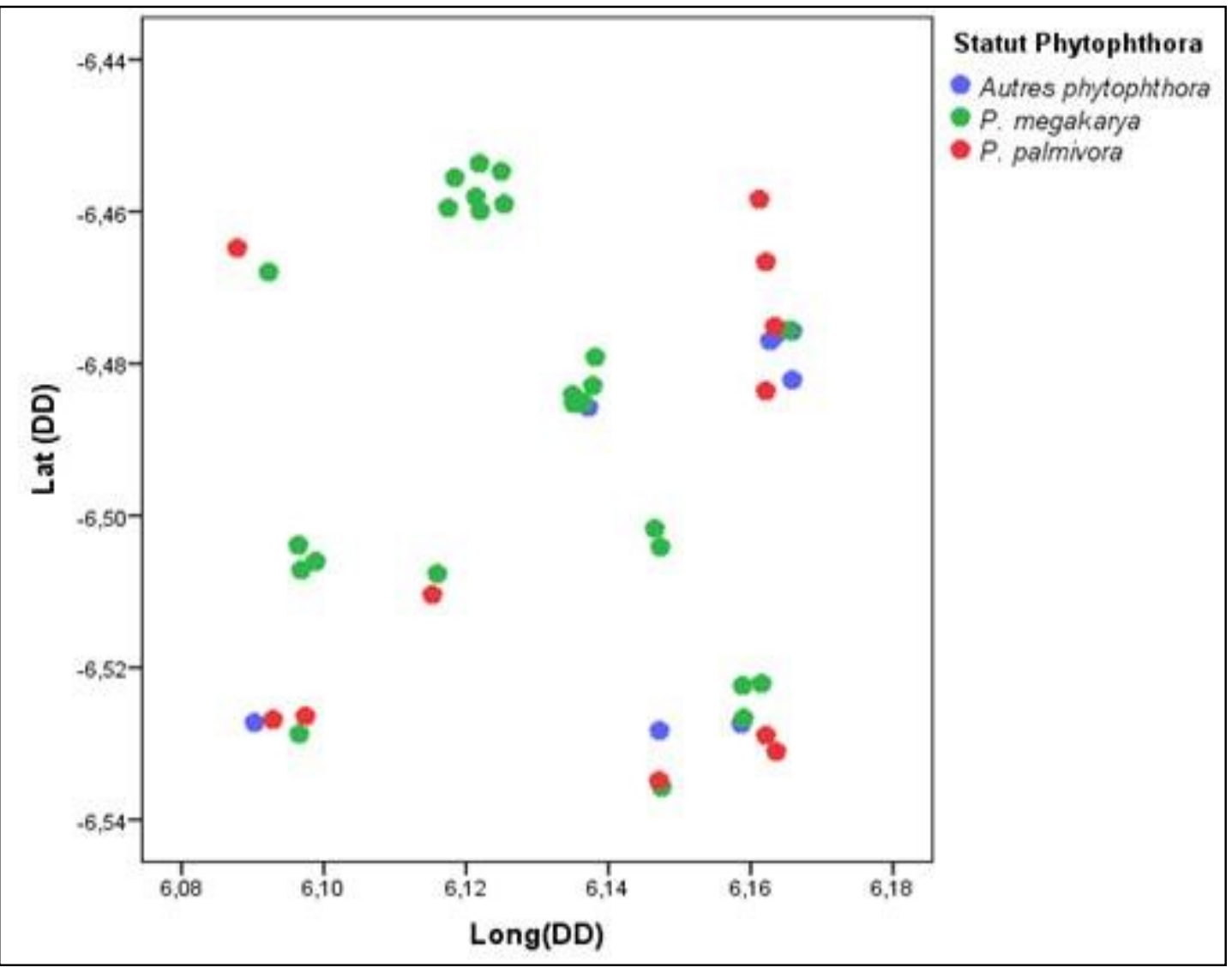

Figure 4: Carte de dispersion des espèces de Phytophthora sp sur le site sentinelle de Kipiri 


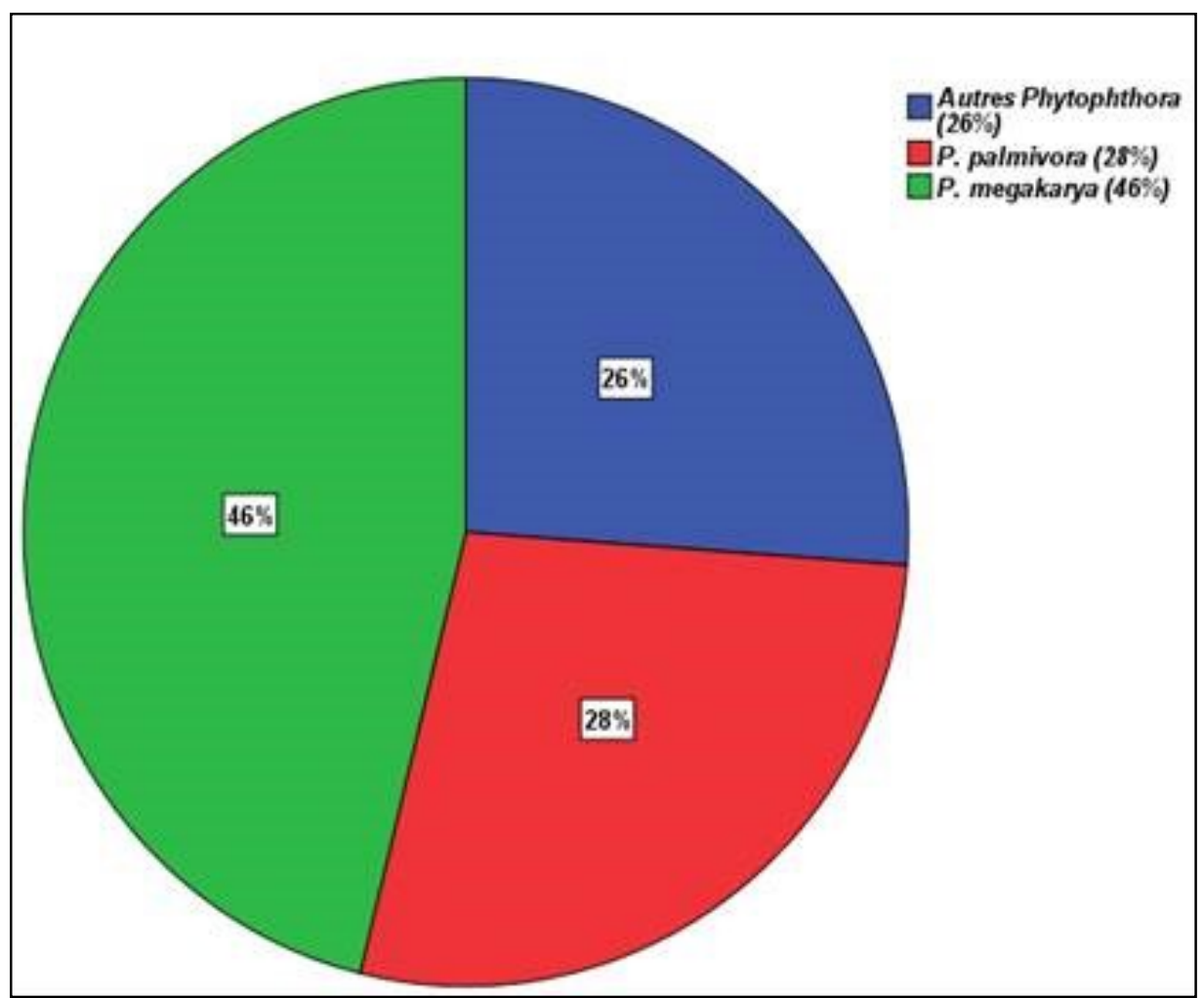

Figure 5 : Diagramme camembert des proportions des différentes espèces de la pourriture brune des cabosses.

Tableau 1: Statistique descriptive de la prévalence de la pourriture brune des cabosses

\begin{tabular}{llllll}
\hline & Minimum & Maximum & Somme & Moyenne & Ecart type \\
\hline Prévalence Phytophthora & $17,3 \%$ & $39,2 \%$ & $362,4 \%$ & 22,65 & $\pm 6,3$ \\
& & & & & \\
\hline
\end{tabular}

Tableau 2 : Statistique descriptive du Niveau d'Entretien (NE) des plots

\begin{tabular}{lll}
\hline NE & Effectifs NE & Pourcentage \\
\hline Non entretenus & 45 & 69,23 \\
Entretenus & 20 & 30,77 \\
\hline Total & 65 & 100 \\
\hline
\end{tabular}




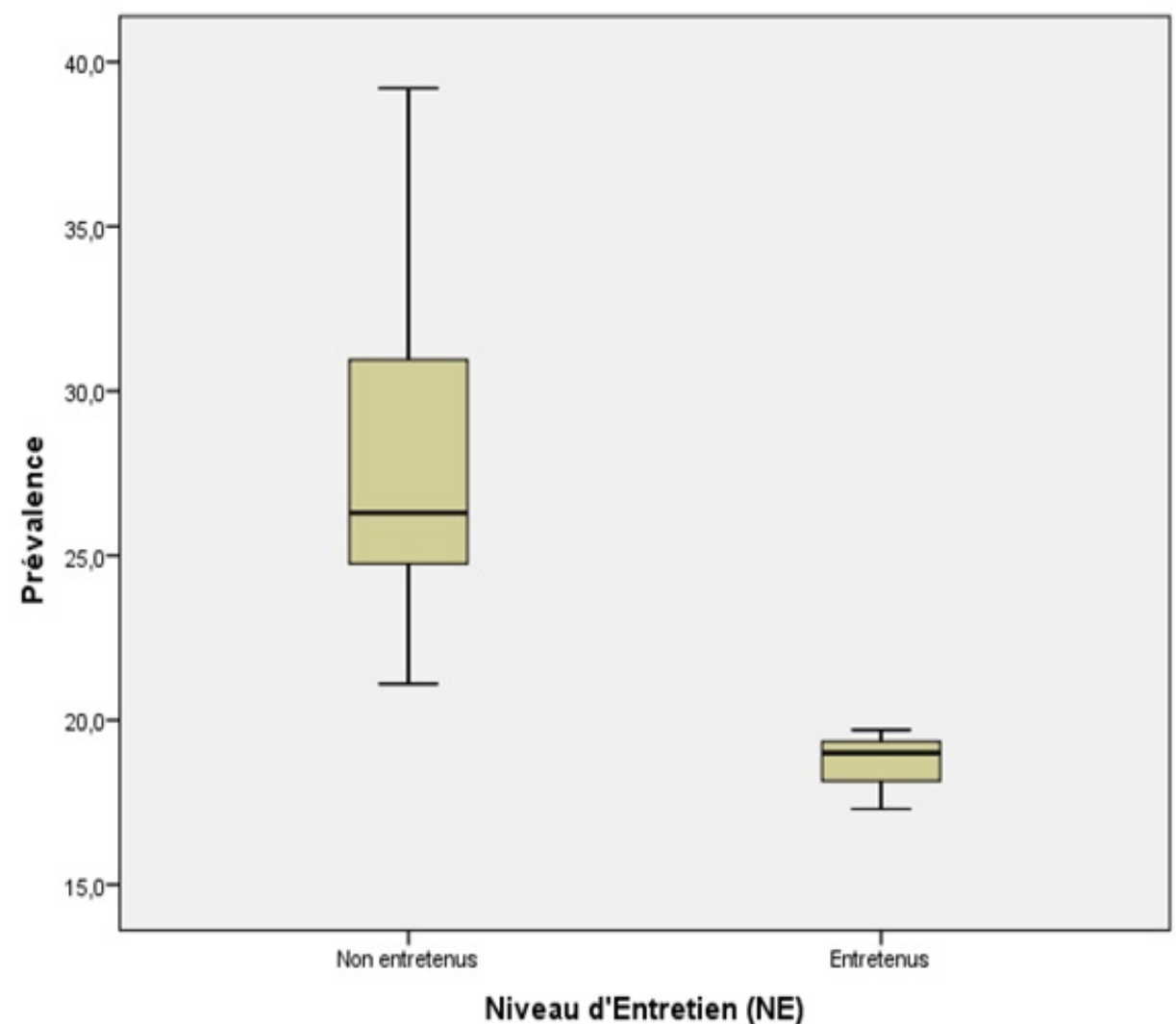

Figure 6 : Boxplots de la prévalence de la pourriture brune en fonction du niveau d'entretien des plots

Tableau 3 : Test de ANOVA à 1 facteur entre le NE des plots et la prévalence de la pourriture brune des cabosses.

\begin{tabular}{llllll|}
\hline & Somme des carrés & dd & Moyenne des carrés & F & Signification \\
\hline Inter-groupes & 198,983 & 1 & 198,983 & 7,55 & $\mathbf{0 , 0 1 8}$ \\
\hline
\end{tabular}

\section{DISCUSSION}

Les résultats de l'analyse moléculaire ont permis d'identifier deux espèces de Phytophthora sp responsables de la pourriture brune des cabosses dans la zone de Kipiri. II s'agit de Phytophthora megakarya à hauteur de $46 \%$ et Phytophthora palmivora à $28 \%$. Les autres espèces de Phytophthora non identifiées représentaient $26 \%$. Ces résultats ont révélé que $P$. megakarya est l'espèce la plus dominante du verger de cacaoyers à Kipiri. La forte présence $P$. megakarya pourrait être la cause de la progression de la pourriture des cabosses à Kipiri. Cela est contraire aux études de Coulibaly et al (2013) qui confirmaient une dominance des Phytophthora palmivora dans les cacaoyères ivoiriennes. En effet, cette espèce de Phytophthora, très virulente, était absente en Côte d'Ivoire, jusqu'à sa dernière découverte en 2000 dans l'Est du pays (Gidoin, 2013). L'expansion de Phytophthora megakarya en plus de celle déjà existante de
Phytophthora palmivora, pourrait bouleverser les systèmes de lutte contre la pourriture brune de cabosses en Côte d'Ivoire avec un risque plus élevé de perte de rendement. Les résultats du test d'ANOVA à 1 facteur ont montré que la prévalence de la pourriture brune des cabosses est dépendante du niveau d'entretien des plots. Cela montre que la recrudescence de la maladie de la pourriture brune dans le département de Soubré est liée au non entretien, voir à l'abandon des parcelles de cacaoyers. En effet, sur l'ensemble des plots d'observations (65 plots), les deux-tiers (45 plots) ne sont pas entretenus soit $69 \%$. Le non entretien de la plantation de cacaoyers favorise le développement de la pourriture brune des cabosses par les insectes ravageurs. Ces insectes une fois dans la parcelle, attaquent les cabosses du cacaoyer pour se nourrir et laisser plusieurs blessures sur les cacaoyers qui constituent 
une porte d'entrée aux Phytophthora sp. Ces résultats sont en accords avec ceux obtenus par Gidoin (2013) au Cameroun. II montre qu'une plantation de cacaoyer non entretenue facilite la propagation de la pourriture brune des cabosses à cause de l'installation des

\section{CONCLUSION ET PERSPECTIVES}

Au terme de cette étude, deux espèces de phytophthora sp ont été identifiées à Kipiri. II s'agit des espèces $P$. megakarya et $P$. Palmivora. L'espèce la plus dominante était $P$. megakarya $(46 \%)$ suivi de l'espèce $P$. Palmivora (28\%). Cependant, il ressort que le niveau d'entretien (NE) des parcelles de cacaoyers a une influence significative sur la prévalence de la pourriture brune des cabosses. En effet les parcelles

\section{REMERCIEMENTS}

Tous nos remerciements à ICRAF, Côte d'Ivoire pour avoir mis les données à notre disposition, ainsi qu'au

\section{REFERENCES BIBLIOGRAPHIQUES}

Babin $R, 2009$. Contribution à l'amélioration de la lutte contre les mirides du cacaoyer Sahlbergella singularis Hagl. (Hemiptera: Miriadae). Influence des facteurs agro-écologiques sur la dynamique des populations du ravageur. Thèse, Université Montpelier III - PAUL VALERIY, $246 \mathrm{p}$.

CCC, 2015. Manuel technique de cacaoculture durable à l'attention du technicien. Manuel, Côte d'Ivoire, $166 \mathrm{p}$.

Coulibaly K., Kébé B., Koffi K., Mpika J., Koné D, 2013. Caractérisation des isolats de Phytophthora spp du verger cacaoyer de Cote d'lvoire. Journal of Applied Biosciences 70:5567- 5579

Coulibaly K., Kébé B., Aka A., Kouakou K., Kassin K., Assi M., Tahi G., 2017. Bien lutter contre la pourriture brune des cabosses du cacaoyer en Côte d'Ivoire. Fiche technique cacaoyer $n^{\circ} 6,2$ $P$.

Diby L., Kouassi G., N'guessan M., Yao E., Oro F., Aynekulu E., Kassin E., Kouamé C., Coe R., Shepherd K, 2014. Cocoa Land Health Surveillance: An evidence-based approach to sustainable management of Cocoa landscapes in the Nawa region, South-West Côte d'Ivoire. Working Paper 193, $34 \mathrm{p}$.

Dufour P, 2005. Le cacao en Côte d'Ivoire. Fiche de synthèse, $4 \mathrm{p}$.

Gidoin C, 2013. Relations entre structure du peuplement végétal et bioagresseurs dans les insectes ravageurs (fourmis, mirides, termites, etc.). Ces insectes accélèrent l'évolution de cette maladie en passant d'une cabosse infectée à une cabosse indemne.

non entretenues étaient plus susceptibles d'être infectées par le Phytophthora sp que les parcelles non infectées. En définitif, le désherbage régulier des parcelles et la taille régulière des cacaoyers et l'ensemble des pratiques d'entretien cultural pourraient constituer un moyen efficace et moins couteux pour lutter contre la maladie de la pourriture brune de cacaoyers.

cabinet d'expertise agricole SAG Sarl pour la mise en œuvre des activités de terrain.

agroforêts à cacaoyers. Application à trois bioagresseurs du cacaoyer : la moniliose au Costa Rica, la pourriture brune et les mirides au Cameroun. Thèse, Centre international d'études supérieures en sciences agronomiques de Montpellier, $210 \mathrm{p}$.

Koua S, 2018. Caractérisation vergers et des maladies de cacao de la Côte d'lvoire: cas des départements d'Abengourou, Divo et Soubré. Journal of Animal \&Plant Sciences, Vol.35, $\mathrm{N}^{\circ} 3$, pp 5706-5714.

Marius W. et Foluke W, 2015. La production de cacao en Afrique de I'Ouest, un examen et une analyse des développements. NJAS Wageningen Journal of Life Sciences. pp 7475.

Ndoumbe N, 2002. Incidence des facteurs agroécologiques sur l'épidémiologie de la pourriture brune des fruits du cacaoyer au Cameroun: Contribution à la mise en place d'un modèle d'avertissements agricoles. Thèse, Institut national agronomique Parisgrignon, $189 \mathrm{p}$.

Ouattara S, 2015. Enjeux de la certification du cacao produit en côte d'ivoire. Revue canadienne de géographie tropicale, Vol. 2, $\mathrm{N}^{\circ} 2$, pp. 43-51.

Tano A, 2012. Crise cacaoyère et stratégies des producteurs de la sous-préfecture de Méadji au Sud-Ouest ivoirien. Thèse, Université de Toulouse, $262 \mathrm{P}$. 